\title{
Thermodynamic Critical Magnetic Field for Chlorine Halide Superconductor at High Pressure
}

\author{
D. SZCZȨŚNIAK ${ }^{a, *}$, R. SZCZȨŚNIAK ${ }^{b}$ \\ ${ }^{a}$ Institute of Physics, Jan Długosz University in Częstochowa, al. Armii Krajowej 13/15, \\ 42-200 Czȩstochowa, Poland \\ ${ }^{b}$ Institute of Physics, Częstochowa University of Technology, al. Armii Krajowej 19, 42-200 Czȩstochowa, Poland
}

\begin{abstract}
At present, hydrides are considered as a one of the most interesting high-temperature superconductors with the classical electron-phonon pairing mechanism. In the present paper, we have analyzed the dependence of the thermodynamic critical magnetic field $\left(H_{c}\right)$ on the temperature for the chlorine halide superconductor. The calculations have been made in the framework of the Eliashberg formalism for the following pressure values: $p_{1}=320 \mathrm{GPa}$ and $p_{2}=360 \mathrm{GPa}$. We have shown that $H_{c}$ increases strongly with the increase of the pressure: $\left[H_{c}(0)\right]_{p_{2}} /\left[H_{c}(0)\right]_{p_{1}}=1.43$. Furthermore, the dimensionless ratio: $R_{H} \equiv T_{c} C^{N}\left(T_{c}\right) / H_{c}^{2}(0)$, where $T_{c}$ and $C^{N}$ denote the critical temperature and the specific heat of the normal state, differs from the value predicted by the Bardeen-Cooper-Schrieffer theory. In particular: $\left[R_{H}\right]_{p_{1}}=0.166$ and $\left[R_{H}\right]_{p_{2}}=0.158$.
\end{abstract}

DOI: 10.12693/APhysPolA.126.344

PACS: 74.20.Fg, 74.25.Bt, 74.62.Fj

In recent years, hydrides have gained much attention as possible pressure-induced high-temperature superconductors with the conventional electron-phonon pairing mechanism $[1,2]$. This interest stems from the fact that, in hydrides, it is theoretically possible to obtain a high critical temperature $\left(T_{c}\right)$ at the pressure $(p)$ which is much lower than the metallization pressure $(\sim 400 \mathrm{GPa})$ for the pure metallic hydrogen $[3,4]$. In certain cases, like for example in the case of $\mathrm{Si}_{2} \mathrm{H}_{6}$, the critical temperature is expected, on the basis of the theoretical calculations [5], to be as high as $173 \mathrm{~K}$ at $p=275 \mathrm{GPa}$. Please note that this value of $T_{C}$ is even higher than for the cuprate superconductor $\mathrm{HgBa}_{2} \mathrm{Ca}_{2} \mathrm{Cu}_{3} \mathrm{O}_{8+y}$, where the maximal critical temperature is equal to $164 \mathrm{~K}$ at $p=31 \mathrm{GPa}[6]$. Thus, it is important to examine the promising superconducting properties of such hydrogen compounds.

In the presented work, we have studied the dependence of the thermodynamic critical magnetic field $\left(H_{C}\right)$ on the temperature for the chlorine halide $(\mathrm{HCl})$ superconductor. Despite of the fact that $\mathrm{HCl}$ contains a smaller number of the $\mathrm{H}$ atoms than the hydrogen-rich materials, the hitherto determined superconducting properties of this material are promising [7].

The analysis of the $\mathrm{HCl}$ compound (within the $P 2_{1} / m$ crystal structure) has been carried out for the pressure values $p_{1}=320 \mathrm{GPa}$ and $p_{2}=360 \mathrm{GPa}$. In the considered cases, the electron-phonon coupling constants $(\lambda)$ are beyond the weak-coupling limit $(\lambda>0.3[8])$ : $\lambda_{p_{1}}=0.68$ and $\lambda_{p_{2}}=0.78$. For this reason, the numerical calculations have been conducted in the framework

*corresponding author; e-mail: d.szczesniak@ajd.czest.pl of the Eliashberg formalism [9].

In the paper, we have assumed that the electronphonon interaction is modeled by the Eliashberg functions originally calculated in [7], whereas the Coulomb pseudopotential $\left(\mu^{\star}\right)$ takes a typical value of 0.1 .

The critical magnetic field at a given temperature can be calculated by using the expression (the CGS unit system) $[9]$ :

$$
\frac{H_{c}}{\sqrt{\rho(0)}}=\sqrt{-8 \pi[\Delta F / \rho(0)]},
$$

where $\rho(0)$ stands for the value of the electron density of states at the Fermi level, and $\Delta F$ denotes the free energy difference between the normal and the superconducting state. The latter one can be calculated as follows:

$$
\begin{aligned}
& \frac{\Delta F}{\rho(0)}=-\frac{2 \pi}{\beta} \sum_{m=1}^{M}\left(\sqrt{\omega_{m}^{2}+\Delta_{m}^{2}}-\left|\omega_{m}\right|\right) \\
& \times\left(Z_{m}^{S}-Z_{m}^{N} \frac{\left|\omega_{m}\right|}{\sqrt{\omega_{m}^{2}+\Delta_{m}^{2}}}\right) .
\end{aligned}
$$

The parameter $\beta$ is given by: $\beta \equiv 1 / k_{B} T$, where $k_{B}$ is the Boltzmann constant. The index $M$ limits the number of the Matsubara frequencies: $\omega_{m} \equiv \frac{\pi}{\beta}(2 m-1)$. The symbol $\Delta_{m}$ is the order parameter, whereas $Z_{m}^{S}$ and $Z_{m}^{N}$ denote the wave function renormalization factors for the superconducting and the normal state, respectively.

The order parameter and the renormalization functions have been determined by solving the imaginary axis Eliashberg equations [9]. These complicated calculations have been made by using the iterative methods presented in the papers [10] and [11]. In the considered case, we have assumed that $M=1100$, in order to ensure the stability of the numerical solutions for $T \geq T_{0} \equiv 5 \mathrm{~K}$.

In the bottom panel of Fig. 1, we have presented the dependence of $\Delta F / \rho(0)$ on the temperature for the 
considered values of the pressure. It has been observed that together with the increase of $p$ the absolute values of the free energy difference strongly increase. This fact can be easily described by the following ratio: $[\Delta F(0)]_{p_{2}} /[\Delta F(0)]_{p_{1}}=2.06$, where $\Delta F(0) \equiv \Delta F\left(T_{0}\right)$.

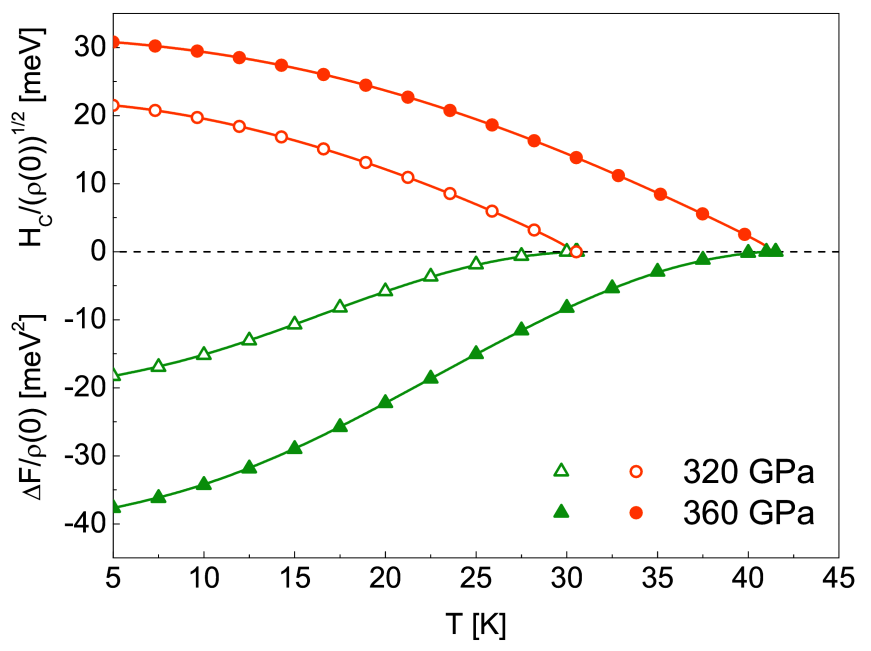

Fig. 1. The dependence of $\Delta F / \rho(0)$ (bottom panel) and $H_{c} / \rho(0)^{1 / 2}$ (upper panel) on the temperature. The results have been presented for two pressure values: $p_{1}=320 \mathrm{GPa}$ (open symbols) and $p_{2}=360 \mathrm{GPa}$ (solid symbols).

The upper panel of Fig. 1 presents the determined dependence of $H_{c} / \rho(0)^{1 / 2}$ on the temperature. It has been shown that the values of the critical magnetic field increase together with the increase of the pressure. This trend is described by: $\left[H_{c}(0)\right]_{p_{2}} /\left[H_{c}(0)\right]_{p_{1}}=1.43$, where $H_{c}(0) \equiv H_{c}\left(T_{0}\right)$.

Next, we have calculated the dimensionless ratio:

$$
R_{H} \equiv \frac{T_{c} C^{N}\left(T_{c}\right)}{H_{c}^{2}(0)},
$$

where the values of the critical temperature for the pressures $p_{1}$ and $p_{2}$ are equal to $30.6 \mathrm{~K}$ and $42.5 \mathrm{~K}$, respectively. The symbol $C^{N}$ denotes the specific heat of the normal state:

$$
\frac{C^{N}(T)}{k_{B} \rho(0)}=\frac{\gamma}{\beta},
$$

where the Sommerfeld constant is given by: $\gamma \equiv \frac{2}{3} \pi^{2}(1+\lambda)$

In the considered case, the numerical calculations have provided the following results: $\left[R_{H}\right]_{p_{1}}=0.166$ and $\left[R_{H}\right]_{p_{2}}=0.158$. It can be observed that the values of $R_{H}$ differ from the prediction of the Bardeen-CooperSchrieffer theory (BCS), where $R_{H}$ is equal to 0.168 $[12,13]$. Therefore, the above fact indicates that the critical magnetic field for the $\mathrm{HCl}$ compound cannot be properly calculated in the framework of the weak-coupling BCS formalism.
In summary, we have determined the form of the function $H_{c}(T)$ for the $\mathrm{HCl}$ superconductor. The following pressure values have been taken into consideration: $320 \mathrm{GPa}$ and $360 \mathrm{GPa}$. The numerical calculations have been conducted in the framework of the Eliashberg formalism. We have shown that the values of the critical magnetic field increase with the increasing pressure: $\left[H_{c}(0)\right]_{p_{2}} /\left[H_{c}(0)\right]_{p_{1}}=1.43$. Moreover, the dimensionless ratio $R_{H}$ differs from the value predicted by the BCS theory: $\left[R_{H}\right]_{p_{1}}=0.166$ and $\left[R_{H}\right]_{p_{2}}=0.158$. The above fact proves that the thermodynamic critical magnetic field for the $\mathrm{HCl}$ compound cannot be determined quantitatively by the weak-coupling BCS model.

\section{Acknowledgments}

The authors are thankful to Z. Ba̧k and K. Dziliński for thier kindness and support throughout this work.

Moreover, authors are grateful to the Czesstochowa University of Technology - MSK CzestMAN for granting access to the computing infrastructure built in the project No. POIG.02.03.00-00-028/08 "PLATON - Science Services Platform".

\section{References}

[1] R. Szczȩśniak, A.P. Durajski, arXiv/1206.5531.

[2] R. Szczȩśniak, A.P. Durajski, Solid State Commun. 153, 26 (2013).

[3] M. Stadele, R.M. Martin, Phys. Rev. Lett. 84, 6070 (2000).

[4] N.W. Ashcroft, Phys. Rev. Lett. 92, 187002 (2004).

[5] R. Szczȩśniak, A.P. Durajski, J. Phys. Chem. Solids 74, 641 (2013).

[6] L. Gao, Y.Y. Xue, F. Chen, Q. Xiong, R.L. Meng, D. Ramirez, C.W. Chu, J.H. Eggert, H.K. Mao, Phys. Rev. B 50, 4260 (1994).

[7] D. Duan, F. Tian, Z. He, X. Meng, L. Wang, C. Chen, X. Zhao, B. Liu, T. Cui, J. Chem. Phys. 133, 074509 (2010).

[8] M. Cyrot, D. Pavuna, Introduction to Superconductivity and High- $T_{c}$ Materials, World Scientific, Singapore 1992.

[9] For the discussion of the Eliashberg formalism [originally formulated by G.M. Eliashberg Sov. Phys. JETP 11, 696 (1960)], we refer to: J.P. Carbotte, Rev. Mod. Phys. 62, 1027 (1990).

[10] R. Szczęśniak, D. Szczȩśniak, Solid State Commun. 152, 779 (2012).

[11] R. Szczęśniak, D. Szczȩśniak, E.A. Drzazga, Solid State Commun. 152, 2023 (2012).

[12] J. Bardeen, L.N. Cooper, J.R. Schrieffer, Phys. Rev 106, 162 (1957).

[13] J. Bardeen, L.N. Cooper, J.R. Schrieffer, Phys. Rev. 108, 1175 (1957). 